\title{
Approaching ballistic transport in suspended graphene
}

\author{
XU DU, IVAN SKACHKO, ANTHONY BARKER AND EVA Y. ANDREI* \\ Department of Physics and Astronomy, Rutgers the State University of New Jersey, 136 Frelinghuysen Rd, Piscataway, New Jersey 08854, USA \\ *e-mail: eandrei@physics.rutgers.edu
}

The discovery of graphene $e^{1,2}$ raises the prospect of a new class of nanoelectronic devices based on the extraordinary physical properties $^{3-6}$ of this one-atom-thick layer of carbon. Unlike two-dimensional electron layers in semiconductors, where the charge carriers become immobile at low densities, the carrier mobility in graphene can remain high, even when their density vanishes at the Dirac point. However, when the graphene sample is supported on an insulating substrate, potential fluctuations induce charge puddles that obscure the Dirac point physics. Here we show that the fluctuations are significantly reduced in suspended graphene samples and we report lowtemperature mobility approaching $200,000 \mathrm{~cm}^{2} \mathrm{~V}^{-1} \mathrm{~s}^{-1}$ for carrier densities below $5 \times 10^{9} \mathrm{~cm}^{-2}$. Such values cannot be attained in semiconductors or non-suspended graphene. Moreover, unlike graphene samples supported by a substrate, the conductivity of suspended graphene at the Dirac point is strongly dependent on temperature and approaches ballistic values at liquid helium temperatures. At higher temperatures, above $100 \mathrm{~K}$, we observe the onset of thermally induced longrange scattering.

The unusual low energy excitation spectrum of graphene, consisting of quasiparticles that are massless Dirac fermions, implies the emergence of novel electronic properties such as negative index of refraction ${ }^{7}$, specular Andreev reflections at graphene-superconductor junctions ${ }^{8,9}$ and evanescent transport ${ }^{10}$. Moreover, because graphene is a semimetal, it can be reduced to nanoscale dimensions without becoming an insulator as occurs in doped semiconductors. Underlying these predictions is the assumption that graphene is minimally affected by interactions with the environment. However, the environment ${ }^{11,12}$, particularly the substrate ${ }^{13}$, can significantly affect such ultrathin films. For graphene deposited on silicon $/ \mathrm{SiO}_{2}$, substrate-induced carrier inhomogeneity due to trapped charges in the oxide or at the graphene-oxide interface is especially deleterious near the Dirac point where screening is weak ${ }^{14-17}$ leading to reduced carrier mobility. In addition, the atomic roughness of the substrate can cause the mobility to deteriorate by introducing short-range scattering centres or by inducing quench-condensation of ripples within the graphene layer ${ }^{18}$. Here we address one of the outstanding questions about graphene-what are its intrinsic transport properties close to the Dirac point-by studying graphene in the absence of a supporting substrate.

To eliminate substrate-induced perturbations, graphene films were suspended from gold/titanium contacts to bridge a trench in a $\mathrm{SiO}_{2}$ substrate (Fig. 1a). In contrast to earlier realizations of suspended graphene $(\mathrm{SG})^{19,20}$, which did not provide electrical contacts for transport measurements, the SG devices described here incorporate multiple electrodes that allow fourlead transport measurements. The SG devices were fabricated from conventional non-suspended graphene (NSG) devices using wet chemical etching (see Supplementary Information). In a typical SG device (Fig. 1b), the graphene layer is suspended from the voltage leads, which run across the sample and at the same time provide structural support. This two-lead configuration avoids complications such as sensitivity to details of the lead geometry that arise in ballistic devices when transport measurements are carried out with a conventional Hall bar ${ }^{21}$ design. For the two-lead voltage configuration used here, the measured transport properties of ballistic devices depend on lead separation and doping in a straightforward way that can be calculated from first principles ${ }^{10}$ (see Supplementary Information).

Low-temperature $(4.2 \mathrm{~K})$ magnetotransport measurements were carried out to determine the number of graphene layers and to obtain the relation $n\left(V_{\mathrm{g}}\right)$ between the induced carrier density $n$ and the gate voltage $V_{\mathrm{g}}$ (Fig. 1c,d,e). Note that $n\left(V_{\mathrm{g}}\right)$ is linear in $V_{g}$, indicating that, within the range of applied gate voltages, bending of the graphene sheet due to the electrostatic force is negligible. Comparing the carrier density capacitance of the SG sample $\alpha_{\mathrm{SG}}=n / V_{\mathrm{g}}\left(\approx 2.14 \times 10^{10} \mathrm{~cm}^{-2} \mathrm{~V}^{-1}\right)$ to that of the NSG sample, $\left(\alpha_{\mathrm{NSG}} \approx 7.4 \times 10^{10} \mathrm{~cm}^{-2} \mathrm{~V}^{-1}\right)$, we find that the ratio $\alpha_{\mathrm{NSG}} / \alpha_{\mathrm{SG}}=3.46$ is $\sim 15 \%$ smaller than the ratio of the corresponding dielectric constants $\left(\mathrm{SiO}_{2}\right.$ and vacuum). This suggests a slight permanent sagging of the SG devices that is attributed to the deformation of the leads by wicking action of liquids during the fabrication process.

We next focus on the carrier density dependence of the resistivity $\rho(n)$ in a zero magnetic field in the temperature range 4.2-250 K (Fig. 2a). The particle-hole asymmetry seen in the figure is observed in all our samples, SG as well as NSG, if the lead separation is sufficiently short $(L<1 \mu \mathrm{m})$, but it becomes negligible in samples with large lead separation or in the samples with the Hall bar geometry. This implies a contact-induced asymmetry that could arise due to contamination during fabrication or doping by the lead material. However, it could also occur as a result of the unique boundary conditions imposed by the two-lead geometry. Further studies are needed to determine the precise cause of the asymmetry. We limit our discussion to the hole branch where well-defined Shubnikov-de-Haas (ShdH) oscillations are observed. For SG samples, the peak in $\rho\left(V_{\mathrm{g}}\right)$ at the Dirac point becomes very sharp at low temperatures. On the hole branch the half-width at half-maximum (HWHM) is almost 

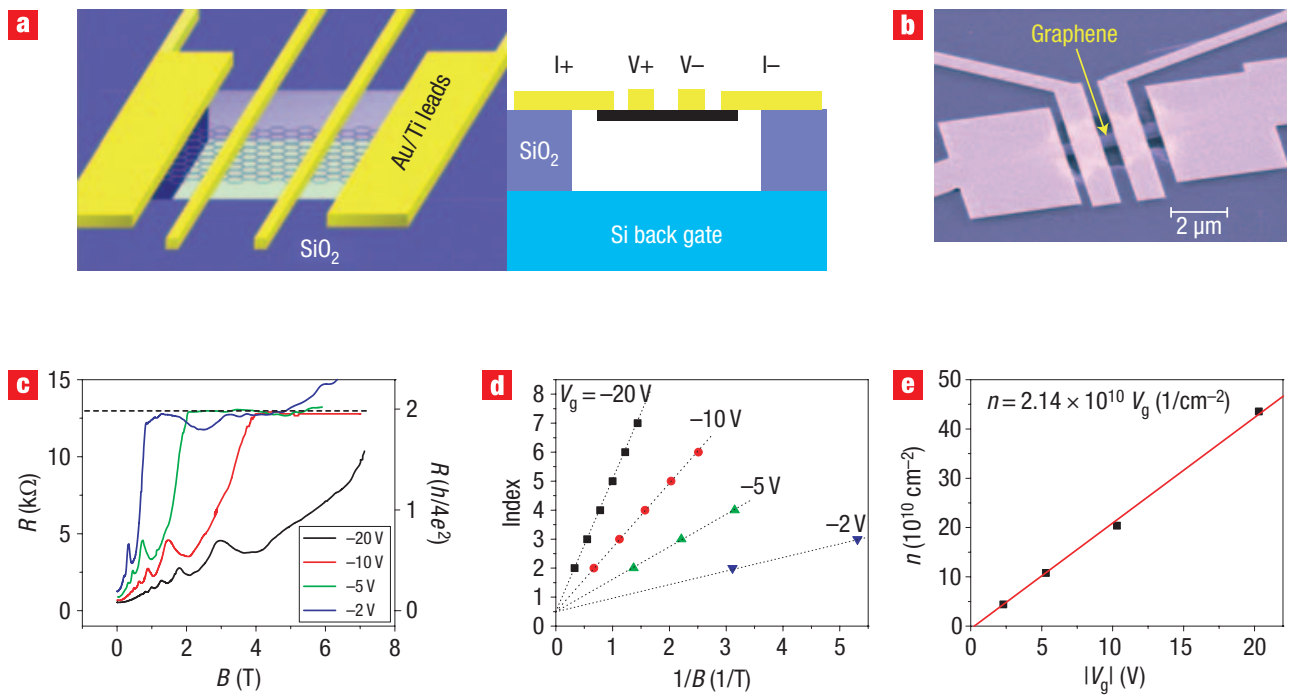

Figure 1 Suspended graphene (SG) sample characterization. a, Schematic representation of the structure of the SG devices: top view (left panel), side view (right panel). The electrodes are shown in yellow and the $\mathrm{SiO}_{2}$ in blue. b, Scanning electron microscope image of a typical SG sample. c, Shubnikov-de-Haas (ShdH) oscillations in the hole branch at the indicated gate voltages. The plateaus at $R_{x x} \approx 13 \mathrm{k} \Omega\left(h / 2 e^{2}\right)$ reflect a contribution from the Hall resistance, seen as a result of the non-ideal shape of the sample. $\mathbf{d}, \mathrm{A}$ 'fan diagram' constructed for the hole branch from the gate dependence of the ShdH oscillations in $\mathbf{c}$ by plotting the Landau level index versus $1 / B$ for the indicated gate voltages $V_{\mathrm{g}}$. The linear dependence extrapolated to the index number $1 / 2$ identifies the sample as single-layer graphene ${ }^{6}$. e, The slopes of the curves in $\mathbf{d}$ give the ShdH oscillation periods $1 / B_{\mathrm{F}}$ for each value of $V_{\mathrm{g}}$, from which the gate dependence of the carrier density $n=4 e / h B_{\mathrm{F}}\left(V_{\mathrm{g}}\right)$ is obtained.

one order of magnitude narrower for SG samples than that of the best NSG samples reported to date $e^{4,22}$. This is directly seen (Fig. 2a) in the side-by-side comparison of the $\rho(n)$ curves for SG and NSG samples taken from the same graphite crystal. In Fig. $2 \mathrm{~b}$ we compare the low-temperature $\rho(n)$ curves with ballistic predictions ${ }^{10}$ for two SG samples (see Supplementary Information). Although the ballistic curve is still sharper than the SG curve, the discrepancy is much smaller than for NSG samples. At low carrier densities, the SG devices show near-ballistic transport away from the Dirac point, as revealed by the weak dependence of the conductance per unit width on channel length (Fig. 2c).

A remarkable feature of the $\rho(n)$ curves in SG samples is the strong temperature dependence of the maximum resistivity, in marked contrast to NSG samples, as illustrated in Fig. 2a. Whereas in NSG the maximum resistivity saturates below $\sim 200 \mathrm{~K}$ (ref. 23), in SG it continues to grow down to much lower temperatures. We attribute this difference to the absence of substrate-induced random potential fluctuations in the SG. The random potential introduces electron-hole puddles ${ }^{15-17}$ close to the Dirac point that cause spatial fluctuations in doping levels. The average deviation of the local Dirac point from the Fermi energy defines an energy bandwidth $E_{\mathrm{F}}^{\mathrm{sat}}$, within which the effect of gating is limited to a redistribution of carriers between electrons and holes without significantly changing the total carrier density. Similarly, the effect of thermally excited electron-hole pairs is masked until $k_{\mathrm{B}} T \approx E_{\mathrm{F}}^{\text {sat }}$ (where $k_{\mathrm{B}}$ is the Boltzmann constant). The effect of the random potential can be estimated (Fig. 3a) from the gate voltage below which the conductivity $\sigma(n)=\rho^{-1}(n)$ no longer changes with gating. This defines a saturation density $n^{\text {sat }}$ that was used to estimate $E_{\mathrm{F}}^{\mathrm{sat}}=\hbar v_{\mathrm{F}}\left(n^{\mathrm{sat}} \pi\right)^{1 / 2}$, where $v_{\mathrm{F}}=1 \times 10^{6} \mathrm{~m} \mathrm{~s}^{-1}$ is the Fermi velocity. In Fig. $3 \mathrm{~b}$ we compare the temperature dependence of $E_{\mathrm{F}}^{\text {sat }}$ for two SG samples and one NSG sample. At high $T$, the slopes of the SG curves approach $k_{\mathrm{B}}$ as expected for thermally excited carriers. At low $T$, where the fluctuation energy is controlled by charge inhomogeneity, its value in the SG samples, $E_{\mathrm{F}}^{\mathrm{sat}}<10 \mathrm{meV}$, is much smaller than in the best NSG samples reported to date, $E_{\mathrm{F}}^{\text {sat }} \approx 40 \mathrm{meV}$ (ref. 22). A direct consequence of the low level of potential fluctuations in the SG samples is that one can follow the intrinsic transport properties of Dirac fermions much closer to the Dirac point than is possible with NSG samples. The effect of temperature on the conductivity curves $\sigma(n)$ is shown in Fig. 3c. Outside the saturation regime, the curves can be fit with a model where scattering is primarily due to boundary confinement and midgap states ${ }^{24}$ $\sigma_{\text {calc }}=\left(\sigma_{\mathrm{mg}}^{-1}+\sigma_{\text {bal }}^{-1}\right)^{-1}$. Here

$$
\sigma_{\text {bal }}=\frac{L}{W} \frac{4 e^{2}}{h} \sum_{n=1} T_{n}
$$

is the ballistic contribution to the conductivity, the summation is over all available longitudinal transport channels and $T_{n}$ is the transmission probability in the $n$th channel, which depends on sample width, lead separation and chemical potential. The Landauer formula is used because, as we show below, the mean free path $(\mathrm{mfp})$ is comparable to the lead separation ${ }^{10}$. Because of interference effects associated with the boundaries, $T_{n}$ (and consequently all other transport properties) is an oscillatory function of the chemical potential ${ }^{10}$ (see Supplementary Information). The other term,

$$
\sigma_{\mathrm{mg}}=\frac{e^{2}}{n_{i} h} \frac{2}{\pi} k_{\mathrm{F}}^{2}\left(\ln k_{\mathrm{F}} R_{0}\right)^{2},
$$

describes scattering by midgap states produced by topological defects of characteristic size $R_{0}$ and density $n_{i}$. The fit at $4 \mathrm{~K}$ gives $R_{0} \approx 3.4 \mathrm{~nm}$ and $n_{i} \approx 1 \times 10^{10} \mathrm{~cm}^{-2}$. Close to the Dirac 

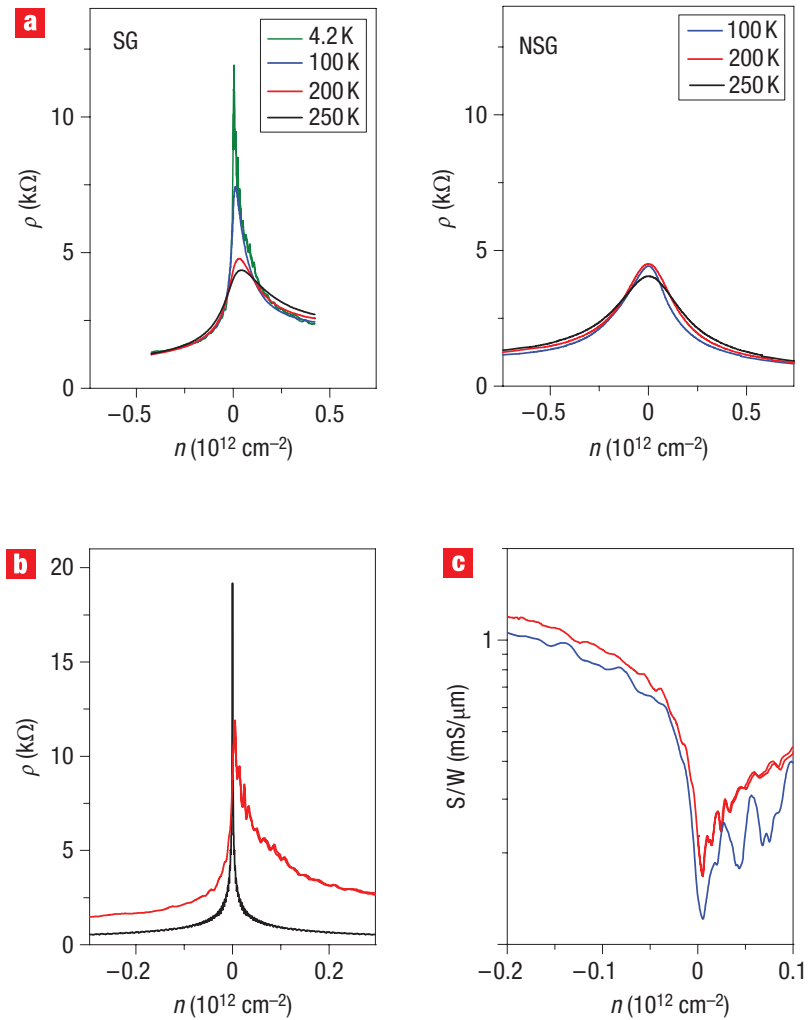

Figure 2 Carrier density dependence of transport. a, Resistivity of an SG device with channel length $L=0.5 \mu \mathrm{m}$ and width $W=1.4 \mu \mathrm{m}$ at the indicated temperatures (left panel). Resistivity of an NSG device with $L=0.5 \mu \mathrm{m}$ (right panel). Note that the resistivity peak almost coincides with the neutrality point $\left(V_{g}=0\right)$, indicating little extrinsic doping. The asymmetry in the curves is observed in all SG samples (see Supplementary Information). The HWHM of the resistivity peak of the $S G$ sample is significantly narrower $\left(\delta V_{\mathrm{g}} \approx 0.15 \mathrm{~V}\right.$, $\left.\delta n \approx 3.2 \times 10^{9} \mathrm{~cm}^{-2}\right)$ than that of the NSG sample $\left(\delta n \approx 2.2 \times 10^{11} \mathrm{~cm}^{-2}\right)$. b. Comparison of the carrier density dependence of the resistivity for the SG device in the left panel of a (red curve, $L=0.5 \mu \mathrm{m}, T=4.2 \mathrm{~K}$ ) with predictions of the ballistic mode ${ }^{10}$ at $T=0$ for channel length $L=0.5 \mu \mathrm{m}$ (black curve). The ballistic model predicts a HWHM of $\delta n \approx 2.14 \times 10^{8} \mathrm{~cm}^{-2}$ for the $\rho(n)$ peak. c, Comparison of conductance per unit width for two SG samples with different dimensions: $L=0.5 \mu \mathrm{m}, W=1.4 \mu \mathrm{m}$ (red curve) and $L=0.25 \mu \mathrm{m}$, $W=0.9 \mu \mathrm{m}$ (blue curve) at $4.2 \mathrm{~K}$.

point the minimum conductivity $\sigma_{0}$ is roughly linear in $T$ for $T<100 \mathrm{~K}$ with a finite intercept at $T=0$ (Fig. 3c, inset),

$$
\sigma_{0}=84 \mu \mathrm{S}=1.7 \frac{4 e^{2}}{\pi h}
$$

Here $4 e^{2} / \pi h$ is the theoretically predicted value at the Dirac point for ballistic transport mediated by evanescent modes ${ }^{10}$. The linear $T$ dependence cannot be understood within existing theoretical models ${ }^{25-27}$. Further work is needed to elucidate how different scattering mechanisms, interactions and the boundary conditions affect the temperature dependence.

We next focus on the mobility, $\mu=\sigma / e n$, and the mean free path, $\mathrm{mfp}=\sigma h /\left(2 e^{2} k_{\mathrm{F}}\right)$, calculated from the measured conductivity. These quantities are meaningful outside the puddle regime, $n>n^{\text {sat }}$ ( $n^{\text {sat }}$ marked by arrows) where the carrier density is known. In Fig. 4a we compare the density dependence
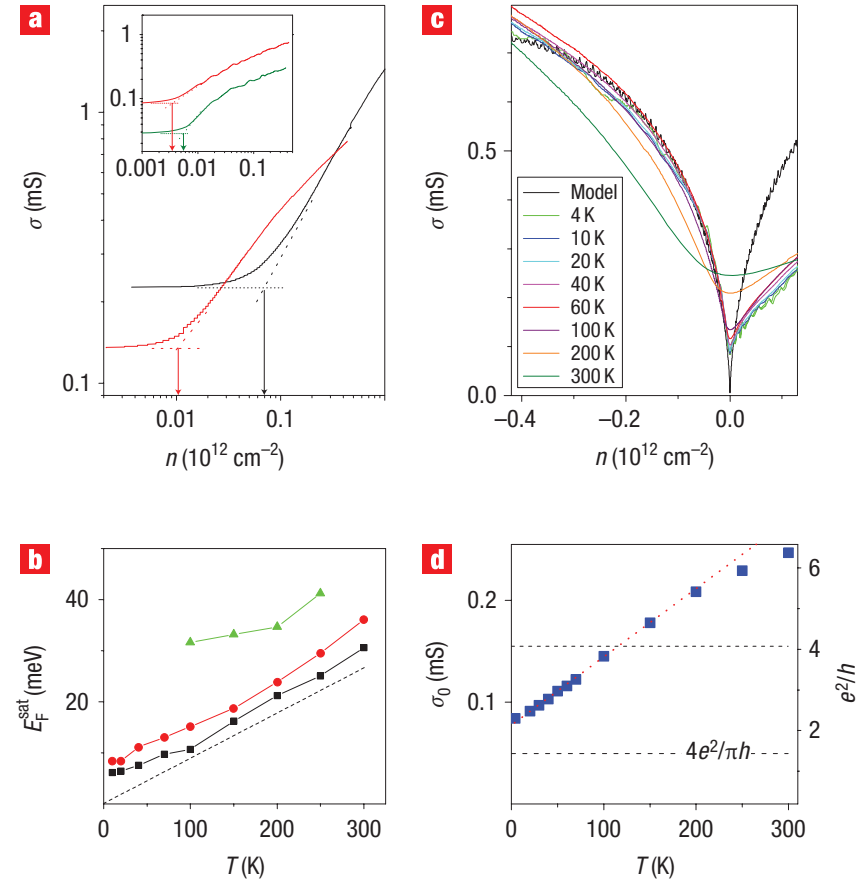

Figure 3 Potential fluctuations. a, Comparison of the carrier density dependence of conductivity for SG (red curve) and NSG (black curve) devices with channel length $L=0.5 \mu \mathrm{m}$, at $T=100 \mathrm{~K}$. The arrows indicate the carrier density below which transport properties are dominated by potential fluctuations. Inset: A similar plot comparing two SG devices: $L=0.5 \mu \mathrm{m}$ (red curve) and $L=0.25 \mu \mathrm{m}$ (green curve) at $T=4.2 \mathrm{~K} . \mathbf{b}$, Temperature dependence of the charge inhomogeneity (measured as the amplitude of the Fermi energy fluctuation) for two SG samples: $L=0.5 \mu \mathrm{m}$ (red), $L=0.25 \mu \mathrm{m}$ (black), and one NSG sample with $L=0.5 \mu \mathrm{m}$ (green). The dotted line represents $E_{\mathrm{F}}^{\text {sat }}=k_{\mathrm{B}} T . \mathbf{c}$, Carrier density dependence of conductivity for the indicated temperatures (the data were shifted to align the minima with the neutrality point). For $T<100$, outside the saturation regime the data collapse onto the calculated curve $\sigma_{\text {calc }}=\left(\sigma_{\mathrm{mg}}^{-1}+\sigma_{\text {bal }}^{-1}\right)^{-1}$, where $\sigma_{\text {bal }}$ is the ballistic contribution controlled by lead confinement ${ }^{10}$ and $\sigma_{\mathrm{mg}}$ is the contribution from midgap states ${ }^{24}$ as discussed in the text. d, Temperature dependence of the minimum conductivity of the device with $L=0.5 \mu \mathrm{m}$ and $W=1.4 \mu \mathrm{m}$. The dashed lines correspond to $4 e^{2} / h$ (upper line) and the theoretical value for evanescent transport $4 e^{2} / \pi h$ (lower line). The dotted line is a guide to the eye.

of the mobility for SG and NSG samples. Just outside the puddle regime, $n \approx 4 \times 10^{9} \mathrm{~cm}^{-2}$ at $T=100 \mathrm{~K}$, the maximum mobility of the SG samples, $\sim 120,000 \mathrm{~cm}^{2} \mathrm{~V}^{-1} \mathrm{~s}^{-1}$, is significantly larger than that measured in the best NSG samples, 2,000$20,000 \mathrm{~cm}^{2} \mathrm{~V}^{-1} \mathrm{~s}^{-1}$. The mobility of the SG samples continues to decrease upon further cooling, reaching its highest value, $\sim 185,000 \mathrm{~cm}^{2} \mathrm{~V}^{-1} \mathrm{~s}^{-1}$, at $T=20 \mathrm{~K}$. Because at low densities the mobility is mostly determined by long-range scattering ${ }^{15}$ (shortrange scattering is weak near the Dirac point due to the small density of states ${ }^{24}$ ), the lower mobility of the NSG samples implies that removal of the substrate eliminates the primary source of long-range scattering. At high carrier densities $\left(n>4 \times 10^{11} \mathrm{~cm}^{-2}\right)$, the mobility in SG and NSG becomes comparable $\left(\sim 10,000 \mathrm{~cm}^{2} \mathrm{~V}^{-1} \mathrm{~s}^{-1}\right)$, suggesting dominant shortrange scattering. Short-range scatterers reflect imperfections in the graphene layer, which may be inherited from the parent graphite crystal or could be introduced during the fabrication process. Similar conclusions can also be reached from the $\operatorname{mfp}(n)$ 

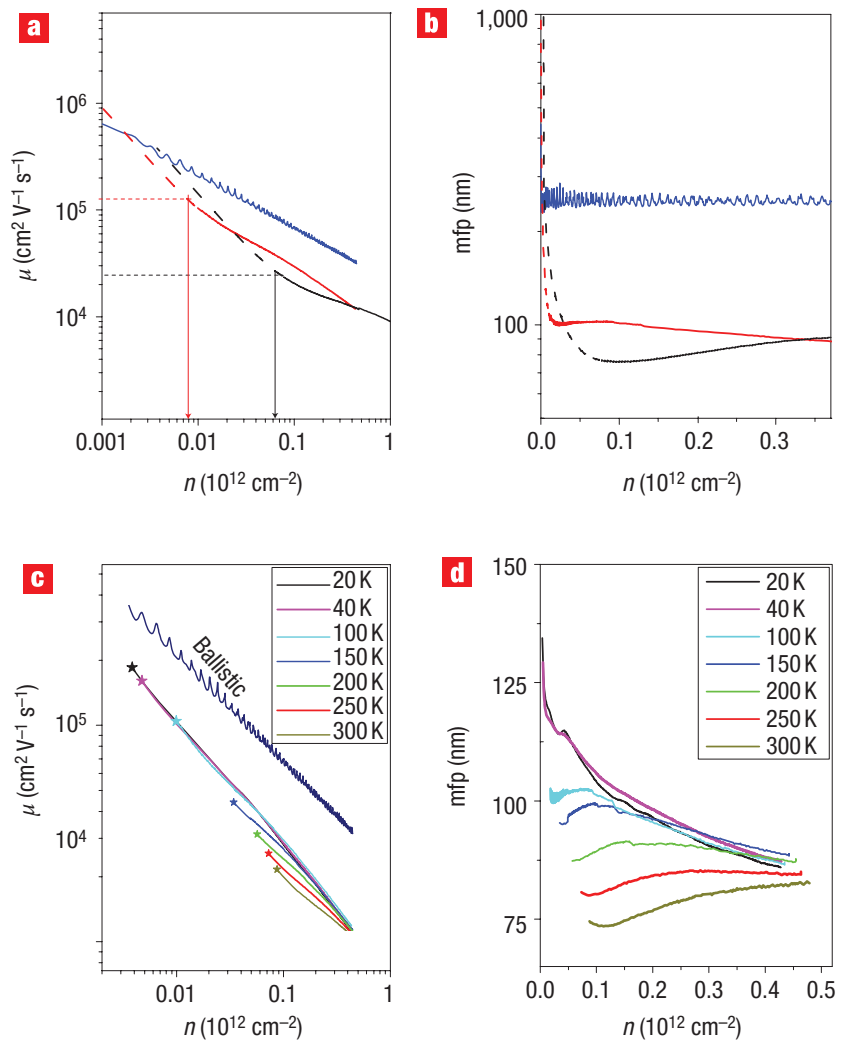

Figure 4 Mobility and mean free path $(\mathrm{mfp})$ of hole branch carriers.

a, Carrier density dependence of mobility for the NSG device(black curve) and the SG device (red curve) at $T=100 \mathrm{~K}$, and ballistic model predictions (blue curve). The onset of the electron-hole puddle regime, $n^{\text {sat }} \approx 6 \times 10^{10} \mathrm{~cm}^{-2}$ and $4 \times 10^{9} \mathrm{~cm}^{-2}$ for the NSG and SG samples, respectively, is indicated by arrows. In the puddle regime $\left(n<n^{\text {sat }}\right)$ the mobility cannot be obtained from these transport measurements, as discussed in the text. For these data, the maximum meaningful mobility is $\sim 120,000 \mathrm{~cm}^{2} \mathrm{~V}^{-1} \mathrm{~s}^{-1}$ and $20,000 \mathrm{~cm}^{2} \mathrm{~V}^{-1} \mathrm{~s}^{-1}$ for the SG and NSG samples, respectively. The finite mobility and oscillatory dependence on carrier density shown in the theoretical curve is a consequence of the finite size imposed by the voltage leads, as discussed in the text. $\mathbf{b}$, Carrier density dependence of the mfp for the NSG (black curve) and SG (red curve) devices at $T=100 \mathrm{~K}$ and the ballistic model prediction (blue curve). c, Carrier density dependence of mobility outside the puddle regime at the indicated temperatures. $\mathbf{d}$, Carrier density dependence of $\mathrm{mfp}$ outside the potential fluctuation regime at the indicated temperatures.

curves in Fig. 4b: the positive slope for the NSG samples reflects long-range scattering ${ }^{15}$, and the negative slope for the SG sample is consistent with the absence of long-range scatterers such as charged impurities ${ }^{15,24}$. This is reinforced by the temperature dependence of the $\operatorname{mfp}(n)$ curves for the SG sample (Fig. 4d). The negative slope and absence of $T$ dependence for $T<100 \mathrm{~K}$ suggest predominantly short-range scattering. Comparing with the ballistic prediction (Fig. $4 \mathrm{~b}$ ), we find that at low $T$ the $\mathrm{mfp}$ in the SG sample is within $\sim 50 \%$ of the ballistic value, which requires a description of the transport in terms of the Landauer formalism. For $T>100 \mathrm{~K}$, the trend to positive slopes suggests the onset of thermally induced long-range scattering, possibly due to thermally excited ripples ${ }^{18}$ or ripple-induced charge inhomogeneity ${ }^{28}$. However, more work is needed to understand the scattering mechanism in this regime.
After completion of this study we became aware of transport measurements on SG samples ${ }^{29}$ carried out with a Hall probe lead geometry. Although the maximum calculated mobility in both experiments is comparable, it is important to realize that the different lead configuration can produce qualitatively different results (see Supplementary Information). The geometry of voltage lead configurations is particularly important in such clean systems as it determines the boundary conditions on the electronic wavefunctions: open for the Hall bar arrangement and closed for the two-lead geometry. Thus, in the latter geometry the finite $\mathrm{mfp}$ imposed by the distance between the voltage leads places an upper bound on the mobility so that even for perfectly ballistic graphene, the measured mobility is finite and depends on carrier density. For a sample with dimensions as used in these experiments $L \times W=0.5 \mu \mathrm{m} \times 1.4 \mu \mathrm{m}$, the maximum ballistic mobility is $\sim 280,000 \mathrm{~cm}^{2} \mathrm{~V}^{-1} \mathrm{~s}^{-1}$ at $n \approx 4 \times 10^{9} \mathrm{~cm}^{-2}$.

The transport measurements reported here show that the maximum resistivity in SG samples $(\sim 12-40 \mathrm{k} \Omega)$ is significantly higher than values reported for NSG samples $(\sim 2-7 \mathrm{k} \Omega)$. These values do not coincide with the theoretical prediction for ballistic transport $\pi h / 4 e^{2}$. However, because the contributions from charge inhomogeneity and scattering have opposite effects, the value of the maximum resistivity cannot in itself be proof of ballistic transport. In contrast, the peakwidth of $\rho(n)$ does provide a reliable yardstick for comparing to ballistic predictions because all scattering mechanisms increase the width. Here we have shown that the resistivity peak in SG devices is more than an order of magnitude sharper than in NSG devices. Together with the high value of the measured mobility, which continues to increase down to the lowest temperatures, these experiments provide strong evidence of near ballistic transport in suspended graphene. They open the door to the realization of a new generation of nanodevices based on the unique transport properties of charge carriers governed by Dirac fermion physics.

Received 4 March 2008; accepted 16 June 2008; published 20 July 2008.

References

1. Novoselov, K. S. et al. Electric field effect in atomically thin carbon films. Science 306, $666-669$ (2004)

2. Berger, C. et al. Ultrathin epitaxial graphite: $2 \mathrm{D}$ electron gas properties and a route toward graphenebased nanoelectronics. J. Phys. Chem. B 108, 19912-19916 (2004).

3. Novoselov, K. S. et al. Two-dimensional gas of massless Dirac fermions in graphene. Nature $\mathbf{4 3 8}$, 197-200 (2005).

4. Geim, A. K. \& Novoselov, K. S. The rise of graphene. Nature Mater. 6, 183-191 (2007).

5. Castro Neto, A. H., Guinea, F., Peres, N.M.R., Novoselov, K.S. \& Geim, A.K. The electronic properties of graphene. Rev. Mod. Phys. Preprint at < http://arxiv.org/abs/0709.1163> (2007).

6. Zhang, Y., Tan, Y.-W., Stormer, H. L. \& Kim, P. Experimental observation of the quantum Hall effect and Berry's phase in graphene. Nature 438, 201-204 (2005).

7. Cheianov, V. V., Fal'ko, V. \& Altshuter, B. L. The focusing of electron flow and a veselago lens in graphene p-n junctions. Science 315, 1252-1255 (2007)

8. Cuevas, J. C. \& Yeyati, A. Levy subharmonic gap structure in short ballistic graphene junctions. Phys. Rev. B 74, 180501 (2006).

9. Beenakker, C. W. J. Specular Andreev reflection in graphene. Phys. Rev. Lett. 97, 067007 (2006).

10. Tworzydlo, J. et al. Sub-Poissonian shot noise in graphene. Phys. Rev. Lett. 96, 246802 (2006).

11. DiCarlo, L. et al. Shot noise in graphene. Phys. Rev. Lett. 100, 156801 (2008).

12. Du, X., Skachko, I. \& Andrei, E. Y. Josephson current and multiple Andreev reflections in graphene SNS junctions. Phys. Rev. B 77, 184507 (2008)

13. Sabio, J. et al. Electrostatic interactions between graphene layers and their environment. Phys. Rev. B 77, 195409 (2008).

14. Cho, S. \& Fuhrer, M. S. Charge transport and inhomogeneity near the minimum conductivity point in graphene. Phys. Rev. B 77, 081402 (2008).

15. Hwang, E. H., Adam, S. \& Das Sarma, S. Das Carrier transport in two-dimensional graphene layers. Phys. Rev. Lett. 98, 186806 (2007).

16. Martin, J. et al. Observation of electron-hole puddles in graphene using a scanning single-electron transistor. Nature Phys. 4, 144-148 (2008).

17. Guinea, F., Katsnelson, M. I. \& Vozmediano, M. A. H. Midgap states and charge inhomogeneities in corrugated graphene. Phys. Rev. B 77, 075422 (2008).

18. Katsnelson, M. I. \& Geim, A. K. Electron scattering on microscopic corrugations in graphene. Phil. Trans. Roy. Soc. A 366, 195-204 (2008).

19. Meyer, J. C. et al. The structure of suspended graphene sheets. Nature 446, 60-63 (2007).

20. Bunch, J. S. et al. Electromechanical resonators from graphene sheets. Science 315, 490-493 (2007). 
21. Datta, S. Electronic Transport in Mesoscopic Systems (Cambridge Univ. Press, Cambridge, New York, 1995)

22. Tan, Y. W. et al. Measurement of scattering rate and minimum conductivity in graphene. Phys. Rev. Lett. 99, 246803 (2007).

23. Morozov, S. V. et al. Giant intrinsic carrier mobilities in graphene and its bilayer. Phys. Rev. Lett. 100 016602 (2008).

24. Stauber, T., Peres, N. M. R. \& Guinea, F. Electronic transport in graphene: A semiclassical approach including midgap states. Phys. Rev. B 76, 205423 (2007).

25. Peres, N. M. R., Guinea, F. \& Castro Neto, A. H. Electronic properties of disordered two-dimensional carbon. Phys. Rev. B 73, 125411 (2006).

26. Mariani, E. \& von Oppen, F. Flexural phonons in free-standing graphene. Phys. Rev. Lett. 100, 076801 (2008)

27. Fritz, L., Schmalian, J., Mueller, M. \& Sachdev, S. Quantum critical transport in clean graphene. arXiv:0802.4289v2 (2008).

28. Brey, L. \& Palacios, J. J. Exchange-induced charge inhomogeneities in rippled neutral graphene. Phys. Rev. B 77, 041403 (2008)
29. Bolotin, K. I. et al. Ultrahigh electron mobility in suspended graphene. Solid State Commun. 146, $351-355$ (2008).

Supplementary Information accompanies this paper at www.nature.com/naturenanotechnology.

\section{Acknowledgements}

We thank G. Li and Z. Chen for discussions, S.W. Cheong and M. Gershenson for use of the atomic force microscope and e-beam equipment, V. Kiryukhin for the highly ordered pyrolytic graphite crystals, F. Guinea, A.H. Castro Neto, A. Balatsky, M. Fogler and D. Abanin for further useful discussions. Our work was supported by DOE DE-FG02-99ER45742; and ICAM.

Author information

Reprints and permission information is available online at http://npg.nature.com/reprintsandpermissions/. Correspondence and requests for materials should be addressed to E.Y.A. 